Ergod. Th. \& Dynam. Sys. (2021), 41, 2382-2396 (c) The Author(s) 2020. Published by

Cambridge University Press. This is an Open Access article, distributed

under the terms of the Creative Commons Attribution licence

(http://creativecommons.org/licenses/by/4.0/), which permits unrestricted re-use,

distribution, and reproduction in any medium, provided the original work is properly

cited.

doi:10.1017/etds.2020.50

\title{
The mapping class group action on SU(3)-character varieties
}

\author{
WILLIAM M. GOLDMAN ${ }^{\circ} \dagger$, SEAN LAWTON $\overbrace{}^{\ddagger}$ and EUGENE Z. XIA ${ }^{\circledR} \S$ \\ $\dagger$ Department of Mathematics, University of Maryland, College Park, MD 20742, USA \\ (e-mail: wmg@umd.edu) \\ $\$$ Department of Mathematical Sciences, George Mason University, \\ 4400 University Drive, Fairfax, VA 22030, USA \\ (e-mail: slawton3@gmu.edu) \\ $\S$ Department of Mathematics, National Cheng Kung University, Tainan 701, Taiwan \\ (e-mail:ezxia@ncku.edu.tw)
}

(Received 12 October 2019 and accepted in revised form 28 April 2020)

Dedicated to the memory of Todd A. Drumm

Abstract. Let $\Sigma$ be a compact orientable surface of genus $g=1$ with $n=1$ boundary component. The mapping class group $\Gamma$ of $\Sigma$ acts on the SU(3)-character variety of $\Sigma$. We show that the action is ergodic with respect to the natural symplectic measure on the character variety.

Key words: character variety, ergodicity, simple closed curves

2020 Mathematics Subject Classification: 22F50, 22D40, 37A25 (Primary)

\section{Introduction}

Let $\Sigma=\Sigma_{g, n}$ be the compact oriented surface of genus $g$ with boundary $\partial \Sigma$ which has $n \geq 1$ components denoted $\partial_{1} \Sigma, \ldots, \partial_{n} \Sigma$. Fix a base point $x_{0}$ on $\Sigma$ and let $\pi=\pi_{1}\left(\Sigma, x_{0}\right)$ denote its fundamental group. Let $\operatorname{Homeo}^{+}(\Sigma, \partial \Sigma)$ be the group of orientation-preserving homeomorphisms of $\Sigma$ which fixes $\partial \Sigma$ pointwise. Define the mapping class group of $\Sigma$ :

$$
\Gamma:=\pi_{0}\left(\text { Homeo }^{+}(\Sigma, \partial \Sigma)\right) .
$$

Alternatively, choose base points $x_{i} \in \partial_{i} \Sigma$ and paths from $x_{0}$ to $x_{i}$. Define $\pi_{1}\left(\partial_{i} \Sigma\right)$ as the cyclic subgroup of $\pi$ corresponding to $\pi_{1}\left(\partial_{i} \Sigma, x_{i}\right)$ and determined by the paths between $x_{0}$ and $x_{i}$. Let $\mathrm{Aut}^{+}(\pi, \partial)$ denote the subgroup of $\operatorname{Aut}(\pi)$ which preserves both the conjugacy classes of the subgroups $\pi_{1}\left(\partial_{i}(\Sigma)\right)$ and the orientation of $\Sigma$. Then $\Gamma$ is isomorphic to the image of $\mathrm{Aut}^{+}(\pi, \partial)$ under the quotient homomorphism

$$
\operatorname{Aut}(\pi) \longrightarrow \operatorname{Out}(\pi):=\operatorname{Aut}(\pi) / \operatorname{lnn}(\pi) .
$$


Let $G$ be an algebraic group over $\mathbb{R}$. Then the set of homomorphisms $\pi \rightarrow G$ enjoys the structure of an $\mathbb{R}$-algebraic set denoted $\operatorname{Hom}(\pi, G)$. Choose conjugacy classes $\mathcal{C}_{i} \subset G$ for each $i=1, \ldots, n$ and let $\mathcal{C}:=\left\{\mathcal{C}_{1}, \ldots, \mathcal{C}_{n}\right\}$. Denote by $\operatorname{Hom}_{\mathcal{C}}(\pi, G)$ the subset of $\operatorname{Hom}(\pi, G)$ comprising homomorphisms which send $\pi_{1}\left(\partial_{i} \Sigma\right)$ to $\mathcal{C}_{i}$.

The group $\operatorname{Inn}(G)$ of inner automorphisms of $G$ acts on $\operatorname{Hom}_{\mathcal{C}}(\pi, G)$ by composition. Denote the resulting relative character variety by

$$
\mathcal{M}_{\mathcal{C}}(G):=\operatorname{Hom}_{\mathcal{C}}(\pi, G) / \operatorname{lnn}(G) .
$$

The group $\operatorname{Aut}^{+}(\pi, \partial)$ acts on $\pi$ and hence on $\operatorname{Hom}_{\mathcal{C}}(\pi, G)$ by composition. Furthermore, the action descends to a $\Gamma$-action on $\mathcal{M}_{\mathcal{C}}(G)$. The moduli space $\mathcal{M}_{\mathcal{C}}(G)$ has an invariant dense open subset $\mathcal{M}_{\mathcal{C}}^{o}(G)$, which is a smooth manifold. If, for example, $G$ is $\mathbb{R}$-reductive (see [Gol84, Gol97]), this subset has an $\Gamma$-invariant symplectic structure $\Omega$. In particular, $\mathcal{M}_{\mathcal{C}}^{o}(G)$ admits a natural smooth $\Gamma$-invariant measure $\mu$.

This paper is part of the general program to understand the dynamics of the action of $\Gamma$ and automorphism groups of free groups on character varieties when the Lie group is compact. Suppose that $K$ is a compact Lie group. In [Gol97], Goldman conjectured that $\Gamma$ acts ergodically on $\mathcal{M}_{\mathcal{C}}(K)$ and showed this to be the case when $K$ is locally a product of SU(2)'s and U(1)'s. In [PX02] and [PX03], the conjecture was proved for $g \geq 2$ and also established for almost all boundary classes when $g=1=n$. In [GX11], Goldman and Xia offered an alternative and simpler proof for the case of $K=\mathrm{SU}(2)$.

In this paper, we consider the case $g=1=n$ with $K=\operatorname{SU}(3)$. Then $\Gamma \cong \operatorname{SL}(2, \mathbb{Z})$ (see [FM12]). In this special case, $\mathcal{M}_{\mathcal{C}}(K)$ is explicitly described by the commutator map

$$
\begin{aligned}
K \times K & \stackrel{\kappa}{\longrightarrow} K \\
(a, b) & \longmapsto[a, b]:=a b a^{-1} b^{-1} .
\end{aligned}
$$

Indeed, $\mathcal{M}_{\mathcal{C}}(K) \cong \kappa^{-1}(\mathcal{C}) / \operatorname{lnn}(K)$ for each conjugacy class $\mathcal{C} \subset K$.

THEOREM 1.1. Let $K=\mathrm{SU}(3)$ and $\Sigma=\Sigma_{1,1}$. The $\Gamma$-action is ergodic on $\mathcal{M}_{\mathcal{C}}(K)$ with respect to the measure $\mu$.

We prove this theorem along the lines of the main results in [GX11]. If $\rho \in$ $\operatorname{Hom}_{\mathcal{C}}(\pi, G)$, we denote its $\operatorname{Inn}(G)$-equivalence class as $[\rho]$. Similarly, if $S \subseteq$ $\operatorname{Hom}_{\mathcal{C}}(\pi, G)$, then the corresponding set of $\operatorname{lnn}(G)$-equivalence classes is denoted by $[S]$. A simple closed curve $\alpha$ on $\Sigma$ defines a function

$$
\begin{aligned}
\mathcal{M}_{\mathcal{C}}(K) \stackrel{\mathrm{t}_{\alpha}}{\longrightarrow} & \mathbb{C} \\
{[\rho] \longmapsto } & \operatorname{Tr}(\rho(\alpha)) .
\end{aligned}
$$

The symplectic structure $\Omega$ together with the real and imaginary parts of $t_{\alpha}$ give rise to Hamiltonian flows. The ring-theoretical results in [Law07] imply that the algebra of Hamiltonian vector fields is infinitesimally transitive. It follows that the group generated by these flows is locally transitive and hence ergodic.

Depending on the choice of $\alpha$, these Hamiltonian flows preserve the sets $[\mathrm{H}(a, b)]$ or $\left[\mathrm{H}^{\prime}(a, b)\right]$ defined in $\$ 5.1$. On the other hand, $\Gamma$ contains the Dehn twist $\tau_{\alpha}$ along $\alpha$. The $\tau_{\alpha}$-action also preserves $[\mathrm{H}(a, b)]$ and is ergodic in almost all $[\mathrm{H}(a, b)]$. It follows 
that a $\mu$-measurable function is invariant under $\tau_{\alpha}$ if and only if it is invariant under the Hamiltonian flows associated with $t_{\alpha}$. By local transitivity, such a measurable function is almost everywhere constant.

1.1. Notation and terminology. Let $G$ be a group. Denote the inner automorphism induced by $A \in G$ by

$$
\begin{aligned}
& G \stackrel{\operatorname{lnn}(A)}{\longrightarrow} G \\
& B \longmapsto A B A^{-1} .
\end{aligned}
$$

The set of conjugacy classes in $G$ equals the quotient $G / \operatorname{lnn}(G)$, and we denote the image of a subset $S \subseteq G$ under the quotient map by [S]. Denote the centralizer of $A$ in $G$ by

$$
G_{A}:=\operatorname{Fix}(\operatorname{lnn}(A))<G,
$$

where $\operatorname{Fix}(S)$ is the set of fixed points of $S \subseteq G$.

Define the commutator map of $G$ :

$$
\begin{aligned}
& G \times G \stackrel{\kappa}{\longrightarrow} G \\
& (A, B) \longmapsto[A, B]:=A B A^{-1} B^{-1} .
\end{aligned}
$$

Suppose further that $G$ is a Lie group with Lie algebra $\mathfrak{g}$, and denote the adjoint representation of $G$ on $\mathfrak{g}$ by Ad. Identify $\mathfrak{g}$ with the Lie algebra of right-invariant vector fields on $G$; then, for any right-invariant vector field $X \in \mathfrak{g}$, the element $\operatorname{Ad}(a)(X)$ equals the image of $X$ under left multiplication by $a$. Denote the centralizer of $a$ in $\mathfrak{g}$ by

$$
\mathfrak{g}_{a}:=\operatorname{Fix}(\operatorname{Ad}(a)) \subseteq \mathfrak{g} .
$$

Denote the trace of a matrix $a$ by $\operatorname{Tr}(a)$ and the $\lambda$-eigenspace of a matrix $a$ by $\operatorname{Eig}_{\lambda}(a)$ for a scalar $\lambda \in \mathbb{C}$.

The notation $K$ and $\mathfrak{k}$ is reserved for compact Lie groups and their Lie algebras, respectively.

If $(M, \Omega)$ is a symplectic manifold and $M \stackrel{f}{\longrightarrow} \mathbb{R}$ is a smooth function, denote its Hamiltonian vector field by $\operatorname{Ham}(f)$. We denote the tangent space to a smooth manifold $M$ at a point $p \in M$ by $T_{p} M$.

When we say a set is closed, we mean it to be closed in the classical topology.

2. Character varieties and the mapping class group

We fix a base point on the boundary of $\Sigma:=\Sigma_{1,1}$. The fundamental group $\pi:=\pi_{1}(\Sigma)$ is isomorphic to the rank 2 free group $F_{2}$ generated by homotopy classes of oriented based loops $\alpha$ and $\beta$. We often do not distinguish elements in $\pi$ from corresponding oriented based loops on $\Sigma$.

We write

$$
\pi=\langle\alpha, \beta, \sigma \mid \kappa(\alpha, \beta)=\sigma\rangle,
$$

where $\sigma$ is the boundary element. In this way, we have

$$
R:=\operatorname{Hom}(\pi, K) \cong K \times K \text { and } \mathcal{M}:=R / K,
$$

where the $K$-action is by conjugation. 
In our case, we have only one boundary circle and we let $\mathcal{C} \subseteq K$ be a conjugacy class and $c \in \mathcal{C}$. Then the relative representation variety and character variety are

$$
R_{c}:=\operatorname{Hom}_{\mathcal{C}}(\pi, K):=\kappa^{-1}(c) \text { and } \mathcal{M}_{c}:=R_{c}(K) / K_{c} .
$$

Again, the $K_{c}$-action is by conjugation. In this way, a representation $\rho \in R_{c}$ corresponds to $(a, b) \in K \times K$ such that $\kappa(a, b)=c$. Notice that $\mathcal{M}_{c}$ is usually and equivalently defined as

$$
\mathcal{M}_{c}=\kappa^{-1}(\mathcal{C}) / K
$$

The space $\mathcal{M}_{c}$ has a natural symplectic structure $\Omega$ [Gol84, Gol97].

The diffeomorphism group of $\Sigma$ (fixing the boundary and hence also the base point) acts on $\pi$ and this action descends to a $\Gamma$-action on $\pi$, fixing the conjugacy class of $\sigma$. This further induces an action

$$
\begin{aligned}
\mathcal{M}_{c} \times \Gamma & \longrightarrow \mathcal{M}_{c} \\
([\rho], \gamma) & \longmapsto[\rho \circ \gamma] .
\end{aligned}
$$

The $\Gamma$-action leaves $\Omega$ and $\mu$ invariant [Gol97]. For any oriented simple closed curve $\alpha$ on $\Sigma$, denote by $\tau_{\alpha}$ the Dehn twist along $\alpha$. The mapping class group $\Gamma$ contains all Dehn twists; indeed, the Dehn twists generate $\Gamma$ (although we do not need this fact). Denote by $\mathcal{S}$ the set of homotopy classes of oriented simple closed curves on $\Sigma$.

\section{Compact Lie groups}

This section reviews well-known facts that are used in the proofs. Generic elements are introduced; these are regular elements which are dense in their maximal tori and provide non-trivial dynamics.

3.1. Regularity. Suppose that $M$ is an irreducible algebraic set over $\mathbb{R}$ or $\mathbb{C}$ and $M^{s} \subset$ $M$ its singular locus. Then $U=M \backslash M^{s}$ is a smooth manifold and Zariski dense in $M$. The smooth structure on $U$ gives rise to the Lebesgue measure class on $U$ and on $M$, by assigning $M^{s}$ to be a null set. We shall always mean this class, which coincides with the measure class discussed in the introduction [Hue95].

Let $G$ be a linear semi-simple algebraic group over $\mathbb{C}$ of rank $r$ and $K<G$ a maximal compact subgroup. The corresponding Lie algebras are denoted by $\mathfrak{k}$ and $\mathfrak{g}$, respectively.

Recall that an element $a \in K$ is regular if $K_{a}$ has dimension $r$. In general, $\operatorname{dim}\left(K_{a}\right) \geq r$ and $K_{a}$ contains a maximal torus of $K$. An element $a \in K$ is regular if and only if $K_{a}$ is a maximal torus (that is, a Cartan subgroup) in $K$.

Recall that an action on a topological space is minimal if every orbit is dense. If $a \in K$, denote the Haar measure on $K_{a}$ by $\mu_{K_{a}}$ and the pushforward $\left(L_{b}\right)_{*} \mu_{a}$ under left multiplication $K_{a} \stackrel{L_{b}}{\longrightarrow} b K_{a}$ by $\mu_{b a}$.

3.2. Genericity. In general, regularity is too weak a notion for dynamical complexity. We introduce a notion of genericity, which is more useful for constructing non-trivial dynamics. 
Let $(a, b) \in K \times K$. Then the cyclic group $\langle a\rangle$ acts on the left coset $b K_{a}$ by

$$
b \zeta \stackrel{a^{n}}{\longmapsto} b \zeta a^{n}
$$

where $n \in \mathbb{Z}$ and $\zeta \in K_{a}$.

Proposition 3.1. Let $a \in K$ be a regular element. For any $b \in K$, the following conditions are equivalent:

- the cyclic group $\langle a\rangle<K_{a}$ is Zariski dense in $K_{a}$;

- the cyclic group $\langle a\rangle<K_{a}$ is dense in $K_{a}$;

- the action of $\langle a\rangle$ on $b K_{a}$ is minimal;

- the action of $\langle a\rangle$ on $\left(b K_{a}, \mu_{b a}\right)$ is ergodic.

In this case, we say that $a$ is generic. The proof of Proposition 3.1 uses standard facts about compact abelian Lie groups, such as the following lemma.

LEMMA 3.2. A cyclic subgroup of $K_{a}$ is dense in the classical topology if and only if it is dense in the Zariski topology.

Proof. Any set that is classically dense is also Zariski dense since the Zariski topology is coarser than the classical topology. We now show the converse. Clearly the cyclic group $\langle a\rangle \subset K_{a}$, and its Zariski closure is also an abelian subgroup. Its closure in the classical topology

$$
H:=\overline{\langle a\rangle}
$$

is a closed abelian subgroup of $K_{a}$. Now every compact linear group is Zariski closed (see Onishchik and Vinberg [OV90, §4.4, Theorem 5, pp. 133-134]). Hence, $H$ is Zariski closed in $K_{a}$. Since $\langle a\rangle$ is Zariski dense in $K_{a}$ and $H \supseteq\langle a\rangle, H=K_{a}$.

Proof of Proposition 3.1. The proof now follows from Lemma 3.2 and the fact that dense subgroups of the torus act minimally (see Katok and Hasselblatt [KH95, §1.4, p. 28]) and ergodically (see Katok and Hasselblatt [KH95, Proposition 4.2.2, p. 147] or Walters [Wal82, Theorem 1.9, p. 30]).

\section{Infinitesimal transitivity and Hamiltonian flows}

In this section, we let $G$ be a semi-simple complex algebraic Lie group. Let $M$ be a symplectic manifold and $M \stackrel{f}{\longrightarrow} \mathbb{R}$ a smooth function. Denote by $\operatorname{Ham}(f)$ the associated Hamiltonian vector field.

Definition 4.1. Let $M$ be a manifold and $\mathcal{F}$ be a set of real smooth $\mathbb{R}$-functions on $M$ such that at $x \in M$, the differentials $d f(x)$, for $f \in \mathcal{F}$, span the cotangent space $T_{x}^{*}(M)$. Then $\mathcal{F}$ is said to be infinitesimally transitive at $x . \mathcal{F}$ is infinitesimally transitive on $M$ if $\mathcal{F}$ is infinitesimally transitive at all $x \in M$.

Proposition 4.2. Let $M$ be a connected symplectic manifold and $\mathcal{F}$ be infinitesimally transitive on $M$. Then the group $\mathcal{H}$ generated by the Hamiltonian flows $\operatorname{Ham}(f)$ of the vector fields $\operatorname{Ham}(f)$, for $f \in \mathcal{F}$, acts transitively on $M$.

Proof. See [GX11, Lemma 3.2]. 
We now briefly review results of Goldman [Gol86], describing the flows generated by the Hamiltonian vector fields by simple closed curves on $\Sigma$. In this case, the local flow of this vector field on $\mathcal{M}_{c}$ lifts to a flow on the representation variety $R_{c}$. Furthermore, this flow admits a simple description [Gol86], as follows.

4.1. Invariant functions and flows on groups. Let $G$ be a semi-simple complex Lie group with Lie algebra $\mathfrak{g}$. Then the adjoint representation Ad preserves a non-degenerate symmetric bilinear form $\left\langle_{-},{ }_{-}\right\rangle$on $\mathfrak{g}$. In the case $G=\operatorname{SL}(n, \mathbb{C})$, this is

$$
\langle X, Y\rangle:=\operatorname{Tr}(X Y) \text {. }
$$

Let $G \stackrel{\mathrm{t}}{\rightarrow} \mathbb{R}$ be a function invariant under the inner automorphisms $\operatorname{lnn}(G)$. Following [Gol86], we describe how t determines a way to associate to every element $x \in G$ a oneparameter subgroup

$$
\zeta^{t}(x)=\exp (t F(x))
$$

centralizing $x$. Given $\mathrm{t}$, define its variation function $G \stackrel{F}{\rightarrow} \mathfrak{g}$ by

$$
\langle F(x), v\rangle=\left.\frac{d}{d t}\right|_{t=0} \mathrm{t}(x \exp (t v))
$$

for all $v \in \mathfrak{g}$. Invariance of $\operatorname{t}$ under $\operatorname{lnn}(G)$ implies that $F$ is $G$-equivariant:

$$
F\left(g x g^{-1}\right)=\operatorname{Ad}(g) F(x) .
$$

Taking $g=x$ implies that the one-parameter subgroup

$$
\zeta^{t}(x):=\exp (t F(x))
$$

lies in the centralizer of $x \in G$.

Intrinsically, $F(x) \in \mathfrak{g}$ is dual (by $\left\langle_{-},{ }_{-}\right\rangle$) to the element of $\mathfrak{g}^{*}$ corresponding to the leftinvariant 1-form on $G$ extending the covector $d f(x) \in T_{x}^{*}(G)$.

4.2. Invariant functions and centralizing one-parameter subgroups. Recall that $\mathcal{S}$ denotes the set of homotopy classes of oriented simple closed curves on $\Sigma$. If $\alpha \in \mathcal{S}$ is an oriented homotopy class of based loops, then $\mathrm{t}_{\alpha}$, the trace function of $\alpha$, is defined as

$$
\begin{aligned}
\operatorname{Hom}(\pi, G) \stackrel{\mathrm{t}_{\alpha}}{\longrightarrow} \mathbb{C} \\
\rho \longmapsto \operatorname{Tr}(\rho(\alpha)) .
\end{aligned}
$$

Since the function $G \stackrel{\operatorname{Tr}}{\longrightarrow} \mathbb{C}$ is $\operatorname{Inn}(G)$-invariant, $\mathrm{t}_{\alpha}$ defines a $\mathbb{C}$-valued function (also denoted by $\mathrm{t}_{\alpha}$ ) on $\mathcal{M}_{c}$. Let

$$
\mathrm{t}_{\alpha}^{R}=\operatorname{Re}\left(\mathrm{t}_{\alpha}\right), \quad \mathrm{t}_{\alpha}^{I}=\operatorname{Im}\left(\mathrm{t}_{\alpha}\right) .
$$

Then $\mathrm{t}_{\alpha}^{R}$ and $\mathrm{t}_{\alpha}^{I}$ define $\mathbb{R}$-valued functions on $\mathcal{M}_{c}$.

Let $\alpha \in \mathcal{S}$ and $\Sigma \mid \alpha$ denote the compact surface obtained by splitting $\Sigma$ along $\alpha$. The two components $\alpha_{ \pm}$of $\partial \Sigma \mid \alpha$ corresponding to $\alpha$ are the preimages of $\alpha \subset \Sigma$ under the quotient mapping $\Sigma \mid \alpha \longrightarrow \Sigma$. The original surface $\Sigma$ may be reconstructed as a quotient space under the identification of $\alpha_{-}$with $\alpha_{+}$. 
The fundamental group $\pi$ can be reconstructed from the fundamental group $\pi_{1}(\Sigma \mid \alpha)$ as an HNN-extension:

$$
\pi \cong\left(\pi_{1}(\Sigma \mid \alpha) \amalg\langle\beta\rangle\right) /\left(\beta \alpha_{-} \beta^{-1}=\alpha_{+}\right) .
$$

A representation $\rho$ of $\pi$ is determined by:

- the restriction $\rho^{\prime}$ of $\rho$ to the subgroup $\pi_{1}(\Sigma \mid \alpha) \subset \pi$; and

- the value $\beta^{\prime}=\rho(\beta)$

which satisfies

$$
\beta^{\prime} \rho^{\prime}\left(\alpha_{-}\right) \beta^{\prime-1}=\rho^{\prime}\left(\alpha_{+}\right) .
$$

Furthermore, any pair $\left(\rho^{\prime}, \beta^{\prime}\right)$, where $\rho^{\prime}$ is a representation of $\pi_{1}(\Sigma \mid \alpha)$ and $\beta^{\prime} \in G$ satisfies (3), determines a representation $\rho$ of $\pi$.

Let $\mathrm{t}=\mathrm{t}_{\alpha}^{R}$ or $\mathrm{t}=\mathrm{t}_{\alpha}^{I}$. Define the twist flow $\xi_{\alpha}^{t}$ associated with $\mathrm{t}$ on $\operatorname{Hom}(\pi, \mathrm{SU}(3))$ :

$$
\xi_{\alpha}^{t}(\rho): \gamma \longmapsto \begin{cases}\rho(\gamma) & \text { if } \gamma \in \pi_{1}(\Sigma \mid \alpha), \\ \rho(\beta) \zeta^{t}\left(\rho\left(\alpha_{-}\right)\right) & \text {if } \gamma=\beta,\end{cases}
$$

where $\zeta^{t}$ is defined in (1). This flow covers the flow generated by Ham(t) on $\mathcal{M}_{c}$ (see [Gol86]).

4.3. Infinitesimal transitivity. Let $\mathfrak{X}$ be the geometric invariant theory (GIT) quotient of $\operatorname{Hom}\left(\mathrm{F}_{2}, \mathrm{SL}(3, \mathbb{C})\right)$ by $\operatorname{Inn}(\mathrm{SL}(3, \mathbb{C}))$. Choose $\alpha, \beta \in \mathcal{S}$ as in $\S 4.2$ to correspond to curves with geometric intersection number 1 (or equivalently a free basis of $F_{2}$ ). Let

$$
\mathcal{S}_{\alpha, \beta}:=\left\{\alpha, \beta, \alpha \beta, \alpha \beta^{-1}, \alpha \beta \alpha^{-1} \beta^{-1}\right\} \subset \mathcal{S}
$$

and $\mathcal{S}_{\alpha, \beta}^{-1}:=\left\{\gamma^{-1}: \gamma \in \mathcal{S}_{\alpha, \beta}\right\}$. Let

$$
\mathcal{F}_{\alpha, \beta}:=\left\{\mathrm{t}_{\gamma}: \gamma \in \mathcal{S}_{\alpha, \beta} \cup \mathcal{S}_{\alpha, \beta}^{-1}\right\}
$$

TheOREM 4.3. (Lawton [Law06, Law07]) The coordinate ring $\mathbb{C}[\mathfrak{X}]$ is generated by $\mathcal{F}_{\alpha, \beta}$.

Since $\operatorname{Tr}\left(A^{-1}\right)=\overline{\operatorname{Tr}(A)}$ for $A \in \operatorname{SU}(3)$, the set $\mathcal{F}_{\alpha, \beta}$ is invariant under complex conjugation.

The relative $\mathrm{SU}(3)$-character variety $\mathcal{M}_{c}$ of $\mathrm{F}_{2}$ embeds in $\mathfrak{X}$ as a real semi-algebraic subset. The regular functions on this $\mathbb{R}$-semi-algebraic set are the real and imaginary parts of the restrictions to $\mathcal{M}_{c}$ of the regular functions on $\mathfrak{X}$.

COROLlary 4.4. The set

$$
\mathcal{F}_{\alpha, \beta}^{\mathbb{R}}:=\left\{\mathrm{t}_{\gamma}^{R}, \mathrm{t}_{\gamma}^{I}: \gamma \in \mathcal{S}_{\alpha, \beta}\right\}
$$

is infinitesimally transitive on $\mathcal{M}_{c}$.

Proof. Given the remarks preceding this corollary, the result follows from Theorem 4.3 and [GX11, Lemma 3.1]. 


\section{The Dehn twists}

Let $\alpha \in \mathcal{S}$ and $\tau_{\alpha} \in \Gamma$ be the corresponding Dehn twist. The fundamental group $\pi$ can be reconstructed from the fundamental group $\pi_{1}(\Sigma \mid \alpha)$ as an HNN-extension as in (2). Then $\tau_{\alpha}$ induces the automorphism $\left(\tau_{\alpha}\right)_{*} \in \operatorname{Aut}(\pi)$ defined by

$$
\left(\tau_{\alpha}\right)_{*}: \gamma \longmapsto \begin{cases}\gamma & \text { if } \gamma \in \pi_{1}(\Sigma \mid \alpha), \\ \gamma \alpha & \text { if } \gamma=\beta .\end{cases}
$$

This further induces the map $\left(\tau_{\alpha}\right)^{*}$ on $\operatorname{Hom}(\pi, G)$ mapping $\rho$ to

$$
(\rho)\left(\tau_{\alpha}\right)^{*}: \gamma \longmapsto \begin{cases}\rho(\gamma) & \text { if } \gamma \in \pi_{1}(\Sigma \mid \alpha), \\ \rho(\gamma) \rho(\alpha) & \text { if } \gamma=\beta .\end{cases}
$$

(See [Gol86]).

5.1. Dehn twists and Hamiltonian twist flows. $\quad$ Let $a:=\rho(\alpha)$ and $b:=\rho(\beta)$. Then

$$
\begin{aligned}
R_{c} & =\{\rho \in \operatorname{Hom}(\pi, K): \kappa(\rho(\alpha), \rho(\beta))=c\} \\
& =\{(a, b) \in K \times K: \kappa(a, b)=c\} .
\end{aligned}
$$

Let

$$
\mathrm{H}(a, b):=\{a\} \times b K_{a}, \quad \mathrm{H}^{\prime}(a, b):=a K_{b} \times\{b\} .
$$

Proposition 5.1. If $(a, b) \in R_{c}$, then $\mathrm{H}(a, b), \mathrm{H}^{\prime}(a, b) \subseteq R_{c}$.

Proof. Suppose that $t \in K_{b}$. Then $a t=t a$ and $t^{-1} a^{-1}=a^{-1} t^{-1}$. Then

$$
k(a, b t)=a(b t) a^{-1}(b t)^{-1}=a b t a^{-1} t^{-1} b^{-1}=a b a^{-1} b^{-1}=k(a, b)=c .
$$

Hence, $(a, b t) \in R_{c}$. The proof of $a K_{b} \times\{b\} \subseteq R_{c}$ is similar.

Proposition 5.2. If $(a, b) \in R_{c}$, then the Hamiltonian flows of the vector fields $\operatorname{Ham}\left(\mathrm{t}_{\alpha}^{R}\right)$ and $\operatorname{Ham}\left(\mathrm{t}_{\alpha}^{I}\right)$ preserve $[\mathrm{H}(a, b)]$. Similarly, $\operatorname{Ham}\left(\mathrm{t}_{\beta}^{R}\right)$ and $\operatorname{Ham}\left(\mathrm{t}_{\beta}^{I}\right)$ preserve $\left[\mathrm{H}^{\prime}(a, b)\right]$.

Proof. This follows from (4) and by exchanging $\alpha$ and $\beta$.

COROLlARY 5.3. If a is generic, then $\left\langle\tau_{\alpha}\right\rangle$ acts ergodically on $\mathrm{H}(a, b)$. If $b$ is generic, then $\left\langle\tau_{\beta}\right\rangle$ acts ergodically on $\mathrm{H}^{\prime}(a, b)$.

Proof. By (5), $\tau_{\alpha}(a, b) \in \mathrm{H}(a, b)$ and $\tau_{\beta}(a, b) \in \mathrm{H}^{\prime}(a, b)$. The corollary then follows from Proposition 3.1.

\section{The case of $K=\mathrm{SU}(3)$}

For the rest of this paper, we denote $\omega:=e^{2 \pi i / 3}$ and the identity transformation by $\mathbb{I}$. In this section, we fix $K=\mathrm{SU}(3)$.

The classification of conjugacy classes of $K$ can be described in terms of the trace function

$$
K \stackrel{\operatorname{Tr}}{\longrightarrow} \mathbb{C} .
$$

Let $\Delta=\operatorname{Tr}(K)$ (see Figure 1$)$. 


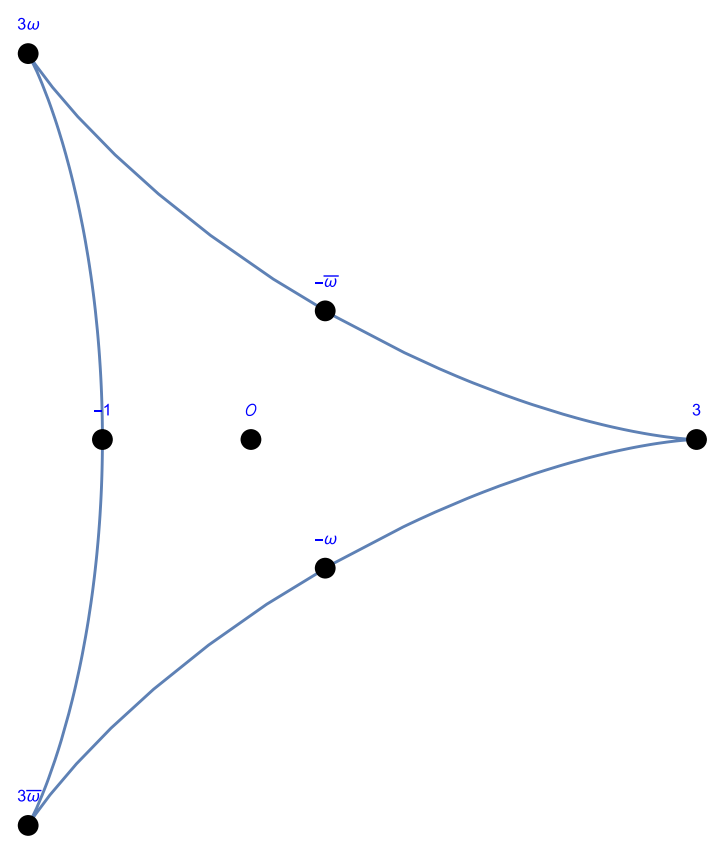

FIGURE 1. $\Delta$, the traces in SU(3).

If $\zeta_{1}, \zeta_{2}, \zeta_{3} \in \mathbb{C}$ are the eigenvalues of $a \in K$, then they satisfy

$$
\left|\zeta_{1}\right|=\left|\zeta_{2}\right|=\left|\zeta_{3}\right|=1 \quad \text { and } \quad \zeta_{1} \zeta_{2} \zeta_{3}=1
$$

The coefficients of the character polynomial $\chi_{a}$ are

$$
\begin{aligned}
1 & =1, \\
\zeta_{1}+\zeta_{2}+\zeta_{3} & =\operatorname{Tr}(a), \\
\zeta_{2} \zeta_{3}+\zeta_{3} \zeta_{1}+\zeta_{1} \zeta_{2} & =\overline{\operatorname{Tr}(a)} \\
\zeta_{1} \zeta_{2} \zeta_{3} & =1 .
\end{aligned}
$$

Therefore, the characteristic polynomial is

$$
\chi_{A}(\lambda)=\lambda^{3}-z \lambda^{2}+\bar{z} \lambda-1
$$

where $z=\operatorname{Tr}(a) \in \mathbb{C}$. Furthermore, (6) is equivalent to the condition

$$
|z|^{4}-8 \operatorname{Re}\left(z^{3}\right)+18|z|^{2}-27 \leq 0
$$

and this real polynomial condition exactly describes the image $\Delta \subseteq \mathbb{C}$.

The traces of central elements are the vertices $3,3 \omega, 3 \bar{\omega}$ of $\Delta$. The trace of a regular element of order three is the center 0 of $\Delta$. The traces of elements of order two are $-1,-\omega,-\bar{\omega}$, the mid-points of the edges of $\partial \Delta$.

PROPOSITION 6.1. The map $\operatorname{Tr}$ is a local submersion at almost all points of $\Delta$. 
Proof. The map $\operatorname{Tr}$ is smooth. Hence, by Sard's theorem, almost all points of $\mathbb{C}$ are regular values of $\operatorname{Tr}[\mathbf{G P 1 0}, \S 1.7]$. Hence, $\operatorname{Tr}$ is a local submersion at almost all points of $\Delta$.

Remark 6.2. It is not difficult to show that $\operatorname{Tr}$ has full rank in the interior of $\Delta$, but we only need Proposition 6.1. For a general discussion of $\Delta$ and Weyl chambers, see [DK00].

Proposition 6.3. The image $\operatorname{Tr}(N)$ of the subset $N \subseteq K$ of generic elements is conull in $\Delta$.

Proof. Let

$$
U=\left\{(\alpha, \beta) \in \mathbb{R} \times \mathbb{R}: \alpha, \beta, \frac{\alpha}{\beta} \in \mathbb{R} \backslash \mathbb{Q}\right\} .
$$

Let $\Delta^{\prime} \subset \Delta$ be the image of $U$ under the mapping

$$
\begin{aligned}
\mathbb{R} \times \mathbb{R} & \longrightarrow \mathbb{C} \\
(\alpha, \beta) & \longmapsto e^{2 \pi i \alpha}+e^{2 \pi i \beta}+e^{-2 \pi i(\alpha+\beta)} .
\end{aligned}
$$

Then $\Delta^{\prime}$ is conull in $\Delta$ and $\operatorname{Tr}^{-1}\left(\Delta^{\prime}\right) \subseteq N$.

7. Central fibers of $\kappa$

Again in this section, we fix $K=\mathrm{SU}(3)$.

7.1. The abelian representations. The fiber $R_{\mathbb{I}}=\kappa^{-1}(\mathbb{I})$ consists of commuting pairs $(a, b)$. In this case, $a$ and $b$ lie in a maximal torus $\mathbb{T}^{2}$. Hence, $\mathcal{M}_{\mathbb{I}} \cong\left(\mathbb{T}^{2} \times \mathbb{T}^{2}\right) / W$, where $W$ is the Weyl group of $K$ acting diagonally (see [FL14] for more discussion of these abelian character varieties).

\subsection{The non-abelian cases.}

Proposition 7.1. If $k=\omega \mathbb{I}$, where $\omega \neq 1$, then $\mathcal{M}_{k}$ consists of a single point. Specifically, if $(a, b) \in R_{k}$, then there exists $g \in K$ such that

$$
a=g a_{0} g^{-1}, \quad b=g b_{0} g^{-1},
$$

where

$$
a_{0}:=\left[\begin{array}{ccc}
1 & 0 & 0 \\
0 & \omega & 0 \\
0 & 0 & \omega^{2}
\end{array}\right], \quad b_{0}:=\left[\begin{array}{ccc}
0 & 0 & 1 \\
1 & 0 & 0 \\
0 & 1 & 0
\end{array}\right] .
$$

Proof. Suppose that $(a, b) \in R_{k}$, that is,

$$
a b a^{-1} b^{-1}=\omega \mathbb{I} \text {. }
$$

We first prove the following lemma.

LEMMA 7.2. $a^{3}=b^{3}=\mathbb{I}$.

Proof of Lemma 7.2. By (8) and taking traces,

$$
\operatorname{Tr}(a)=\operatorname{Tr}\left(\omega b a b^{-1}\right)=\omega \operatorname{Tr}(a) \text { and } \operatorname{Tr}\left(a^{-1}\right)=\operatorname{Tr}\left(\omega b^{-1} a^{-1} b\right)=\omega \operatorname{Tr}\left(a^{-1}\right) .
$$


Hence, $\omega \neq 1$ implies that $\operatorname{Tr}(a)=\operatorname{Tr}\left(a^{-1}\right)=0$. Now apply the Cayley-Hamilton theorem:

$$
a^{3}-\mathbb{I}=a^{3}-\operatorname{Tr}(a) a^{2}+\operatorname{Tr}\left(a^{-1}\right) a-\operatorname{Det}(a) \mathbb{I}=0 .
$$

The same argument applied to $b=\omega a^{-1} b a$ implies that $b^{3}=\mathbb{I}$, as claimed.

Returning to the proof of Proposition 7.1, Lemma 7.2 implies that $a=\operatorname{lnn}(g) a_{0}$ for some $g \in K$, since $a \neq \mathbb{I}$.

We claim that $b=\operatorname{lnn}(g) b_{0}$. For convenience, assume that $a=a_{0}$; then we show that (8) implies that $b$ is the permutation matrix $b_{0}$ defined in (7).

Recall that $\operatorname{Eig}_{\lambda}(a)$ is the $\lambda$-eigenspace of $a$. We claim that (8) implies that

$$
b\left(\operatorname{Eig}_{\lambda}(a)\right)=\operatorname{Eig}_{\omega \lambda}(a)
$$

To see this, rewrite (8) as

$$
a b=\omega b a .
$$

Suppose that $v \in \operatorname{Eig}_{\lambda}(a)$, that is,

$$
a v=\lambda v
$$

so, applying (10),

$$
a(b v)=\omega b a v=\omega \lambda(b v),
$$

whence $b v \in \operatorname{Eig}_{\omega \lambda}(a)$, as claimed.

Since $a$ is the diagonal matrix $a_{0}$ defined by (7), the lines $\operatorname{Eig}_{1}(a), \operatorname{Eig}_{\omega}(a)$ and $\operatorname{Eig}_{\bar{\omega}}(a)$ are the three coordinate lines in $\mathbb{C}^{3}$. Thus, (9) implies that $b=b_{0}$, concluding the proof of Proposition 7.1.

In particular, both $a$ and $b$ have order 3 . Since $\kappa(a, b)$ has order 3 and is central, the subgroup $\langle a, b\rangle \subset K$ is a non-abelian group of order 27 , a non-trivial central extension of $\mathbb{Z} / 3 \oplus \mathbb{Z} / 3$ by $\mathbb{Z} / 3$.

\section{Ergodicity}

Let $K$ be any compact Lie group. Each tangent space $T_{a} K$ identifies with the Lie algebra $\mathfrak{k}$ of right-invariant vector fields: namely, a tangent vector $v \in T_{a} K$ identifies with the rightinvariant vector $X \in \mathfrak{k}$ such that $X(a)=v$. In this way, the differential of the commutator map $\kappa$ at $(a, b) \in K \times K$ identifies with the linear map (see [Gol84, Gol20, PX02]):

$$
\begin{aligned}
& \mathrm{D} \kappa_{(a, b)}: \mathfrak{k} \oplus \mathfrak{k} \longrightarrow \mathfrak{k} \\
&(X, Y) \longmapsto \operatorname{Ad}(b a)\left(\operatorname{Ad}\left(b^{-1}\right)-\mathbb{I}\right) X \\
&+\left(\mathbb{I}-\operatorname{Ad}\left(a^{-1}\right)\right) Y .
\end{aligned}
$$

From this formula, the following proposition holds.

PROPOSITION 8.1. $\kappa$ is submersive at $(a, b)$ if and only if $\mathfrak{k}_{a} \cap \mathfrak{k}_{b}=0$.

For the rest of this section, let $K=\mathrm{SU}(3)$, which comes with the standard representation $\Pi$ on $\mathbb{C}^{3}$. An element in $(a, b) \in R_{c}$ corresponds to a representation $\rho$ of $\pi$ (see $\S 2$ ). Hence, $\rho \circ \Pi$ is a representation of $\pi$. Denote by $\mathcal{M}_{c}^{i} \subseteq \mathcal{M}_{c}$ the subspace of irreducible representation classes. 
8.1. Relation to the moduli space of $K$-bundles. If we fix a complex structure on $\Sigma$, then we can construct the coarse moduli space $\mathcal{N}^{s s}$ of semi-stable parabolic $K$-bundles [BR89] on $\Sigma$, endowed with a complex structure. $\mathcal{N}^{s s}$ contains the subspace $\mathcal{N}^{s}$ of stable parabolic $K$-bundles.

PROPOSITION 8.2. The set of smooth points of $\mathcal{M}_{c}$ is a connected manifold.

Proof. There is a homeomorphism $\mathcal{M}_{c} \cong \mathcal{N}^{s s}$, restricting to a diffeomorphism $\mathcal{M}_{c}^{i} \cong \mathcal{N}^{s}$ [BR89, Theorem 1]. The moduli space $\mathcal{N}^{s s}$ is irreducible and contains $\mathcal{N}^{s}$ as an open subvariety [BR89, Theorem II]. Hence, $\mathcal{N}^{s}$ is open and connected. Hence, $\mathcal{M}_{c}^{i}$ is open and connected. Proposition 8.1 implies that a point $[\rho]=[(a, b)] \in \mathcal{M}_{c}$ is a smooth point if and only if $\rho$ is irreducible, i.e. $\mathcal{M}_{c}^{i}$ is also the set of smooth points of $\mathcal{M}_{c}$. The proposition follows.

For almost every conjugacy class $c$, the action $\mathcal{M}_{c} \times \Gamma \rightarrow \mathcal{M}_{c}$ is ergodic [PX02]. This section proves that this is true for all $c$.

Let $c \in K$. Up to conjugation,

$$
c=\left[\begin{array}{ccc}
c_{1} & 0 & 0 \\
0 & c_{2} & 0 \\
0 & 0 & c_{3}
\end{array}\right]
$$

For $(a, b) \in R_{c}$, we have the natural map

$$
\iota: K_{a} \longrightarrow \mathrm{H}(a, b), \quad \iota(t)=(a, b t) .
$$

Let $P_{2}: K \times K \longrightarrow K$ be the projection to the second factor. An element $\rho \in R_{c}$ corresponds to a pair $(a, b) \in K \times K$. Let

$$
\mathrm{T}=\operatorname{Tr} \circ P_{2}: R_{c} \longrightarrow \Delta \text { and } T:=\iota \circ \mathrm{T}: K_{a} \longrightarrow \Delta .
$$

Proposition 8.3. Let $c \notin Z(K)$. Then $(a, b) \in R_{c}$ exists such that:

(1) $(a, b)$ is a smooth point;

(2) $T$ is a submersion at $(a, b)$.

Proof. Let $(a, b) \in R_{c}$ be such that

$$
a=\left[\begin{array}{lll}
0 & 1 & 0 \\
0 & 0 & 1 \\
1 & 0 & 0
\end{array}\right], \quad b=\left[\begin{array}{ccc}
b_{1} & 0 & 0 \\
0 & b_{2} & 0 \\
0 & 0 & b_{3}
\end{array}\right] .
$$

Then

$$
c=\kappa(a, b)=\left[\begin{array}{ccc}
\frac{b_{2}}{b_{3}} & 0 & 0 \\
0 & \frac{b_{3}}{b_{1}} & 0 \\
0 & 0 & \frac{b_{1}}{b_{2}}
\end{array}\right] .
$$

This formula for $c$ implies that $\kappa$ is onto $K$.

Remark 8.4. A result of Gotô [Got49] states that for any compact semi-simple Lie group $K, K \times K \stackrel{\kappa}{\longrightarrow} K$ is surjective. Hence, $R_{c} \neq \emptyset$ for all $c \in K$. 
Note that $a$ is regular. For (1), there are three cases for $b \in Z(K), b_{1}=b_{2} \neq b_{3}$ (and its permutation variation) and $b$ being regular.

If $b \in Z(K)$, then $\kappa(a, b)=\mathbb{I}=c$ and this violates our hypothesis of $c \notin Z(K)$.

If $b$ is regular, then $t \in \mathfrak{k}_{b}$ implies that

$$
t=\left[\begin{array}{ccc}
t_{1} & 0 & 0 \\
0 & t_{2} & 0 \\
0 & 0 & t_{3}
\end{array}\right], \quad t_{1}+t_{2}+t_{3}=0
$$

Then

$$
(\operatorname{Ad}(a)-\mathbb{I}) t=\left[\begin{array}{ccc}
t_{2}-t_{1} & 0 & 0 \\
0 & t_{3}-t_{2} & 0 \\
0 & 0 & t_{1}-t_{3}
\end{array}\right]
$$

Hence, if $t \in \mathfrak{k}_{a}$, then $(\operatorname{Ad}(a)-\mathbb{I}) t=0$. This implies that $\mathfrak{k}_{a} \cap \mathfrak{k}_{b}=0$. Hence, by Proposition 8.1, we conclude that $\kappa$ is regular at $(a, b)$.

If $b_{1}=b_{2} \neq b_{3}$ and $t \in \mathfrak{k}_{b}$, then

$$
t=\left[\begin{array}{ccc}
t_{11} & t_{12} & 0 \\
t_{21} & t_{22} & 0 \\
0 & 0 & t_{33}
\end{array}\right], \quad t_{1}+t_{2}+t_{3}=0
$$

Then

$$
(\operatorname{Ad}(a)-\mathbb{I}) t=\left[\begin{array}{ccc}
t_{22}-t_{11} & -t_{12} & t_{21} \\
-t_{21} & t_{33}-t_{22} & 0 \\
t_{12} & 0 & t_{11}-t_{33}
\end{array}\right]
$$

Hence, if $t \in \mathfrak{k}_{a}$, then $(\operatorname{Ad}(a)-\mathbb{I}) t=0$. This implies that $t=0$. Hence, $\mathfrak{k}_{a} \cap \mathfrak{k}_{b}=0$. By Proposition 8.1, $\kappa$ is regular at $(a, b)$. We conclude that in all cases $(a, b) \in R_{c}$ is a smooth point.

Notice that $\mathrm{H}(a, b) \subseteq R_{c}$. We consider $T$ restricted to $\mathrm{H}(a, b)$. Let

$$
p=\left[\begin{array}{ccc}
1 & \omega^{2} & \omega \\
1 & \omega & \omega^{2} \\
1 & 1 & 1
\end{array}\right] \text { and } q=\left[\begin{array}{ccc}
1 & 0 & 0 \\
0 & \omega^{2} & 0 \\
0 & 0 & \omega
\end{array}\right] \text {. }
$$

Then $a=p q p^{-1}$. The element $t \in K_{a}$ has the form $t=p t_{b} p^{-1}$, where $t_{b} \in K_{b}$ is diagonal. Then

$$
T(t)=\operatorname{Tr}(b t)=\frac{1}{3} \operatorname{Tr}(b) \operatorname{Tr}(t) .
$$

By Proposition 6.1, $T$ is a local submersion for almost all $t \in \Delta$ unless $\operatorname{Tr}(b)=0$. However, $\operatorname{Tr}(b)=0$ implies that $c \in Z(K)$, which is a contradiction. Hence, $\operatorname{Tr}(b) \neq 0$ and $T$ is a local submersion for almost all $t$.

COROLlARY 8.5. T is a local submersion for almost all points in $\mathrm{H}(a, b)$.

Proof. Since $\mathrm{D} T=\mathrm{DT} \circ \mathrm{D} \iota, \mathrm{D} T_{t}$ being surjective implies that $\mathrm{DT}_{(a, b t)}$ is surjective.

COROLLARY 8.6. There is a conull set $V \subset R_{c}$ such that $b$ is generic for almost all $(a, b) \in V$. 
Proof. The subset containing points at which a map is locally submersive is Zariski open. Hence, by Proposition 8.3, there exists a smooth and Zariski open $V \subseteq R_{c}$ such that $\left.T\right|_{V}$ is a submersion. Let $Q \subseteq R_{c}$ be the smooth part. By Proposition 8.2, $Q$ is connected and hence irreducible. Since a Zariski open subset of a smooth irreducible variety is conull in the Lebesgue class, $V$ is conull. The map $\left.T\right|_{V}$ is a fibration over an open domain of $\Delta$. The corollary follows from Proposition 6.3.

Corollary 8.7. Suppose that $c \notin Z(K)$. Suppose that $\beta \in \mathcal{S}$ and $\phi: \mathcal{M}_{c} \longrightarrow \mathbb{R}$ is a $\mu$-measurable function. If $\phi$ is $\tau_{\beta}$-invariant, then $\phi$ is $\operatorname{Ham}\left(\mathrm{t}_{\beta}^{R}\right)$-invariant and $\operatorname{Ham}\left(\mathrm{t}_{\beta}^{I}\right)$ invariant.

Proof. By Proposition 8.3, $R_{c}$ contains a smooth point $(a, b)$ with $b$ generic. By Proposition 8.2 and Corollary 8.6, $b \in K$ is generic for almost all $(a, b) \in R_{c}$. Hence, $b \in K$ is generic for almost all $[(a, b)] \in \mathcal{M}_{c}$. By Proposition 3.1, 5.1 and Corollary 5.3, $\tau_{\beta}$-orbit is dense in $\mathrm{H}^{\prime}(a, b)$ and $\phi$ is $\operatorname{Ham}\left(\mathrm{t}_{\beta}^{R}\right)$-invariant and $\operatorname{Ham}\left(\mathrm{t}_{\beta}^{I}\right)$-invariant.

With the notation we have adopted, we restate Theorem 1.1 as follows.

THEOREM 8.8. The action $\mathcal{M}_{c} \times \Gamma \rightarrow \mathcal{M}_{c}$ is ergodic.

Proof. Suppose that $c=\mathbb{I}$. Identifying $\mathbb{R}^{2}$ with its dual $\left(\mathbb{R}^{2}\right)^{*}$, the group $\operatorname{SL}(2, \mathbb{Z})$ has the standard dual linear action on $\mathbb{R}^{2}$ which induces the diagonal action on $\mathbb{T}^{2} \times \mathbb{T}^{2}$. This $\mathrm{SL}(2, \mathbb{Z})$-action is known to be ergodic because $\mathrm{SL}(2, \mathbb{Z})$ contains hyperbolic elements, meaning that the eigenvalues of these elements do not have absolute value 1 [BS15, §4].

There is an isomorphism [FM12] $\iota: \Gamma \stackrel{\cong}{\longrightarrow} \operatorname{SL}(2, \mathbb{Z})$. By $\S 7.1, \mathcal{M}_{c} \cong\left(\mathbb{T}^{2} \times \mathbb{T}^{2}\right) / W$. The $\Gamma$-action on $\mathcal{M}_{c}$ lifts to an action on $\mathbb{T}^{2} \times \mathbb{T}^{2}$. Moreover, this $\Gamma$-action is equivariant with respect to $\iota$. Hence, the $\Gamma$-action on $\mathcal{M}_{c}$ is ergodic.

Suppose that $c \in Z(K)$ and $c \neq \mathbb{I}$; then $\mathcal{M}_{c}$ is a single point by Proposition 7.1 and the statement is trivially true.

Suppose that $c \notin Z(K)$. Recall that $\mathcal{H}$ is the group generated by all Hamiltonian flows $\operatorname{Ham}\left(\mathrm{t}_{\beta}^{R}\right)$ and $\operatorname{Ham}\left(\mathrm{t}_{\beta}^{I}\right)$, where $\beta \in \mathcal{S}$. Let $\phi: \mathcal{M}_{c} \longrightarrow \mathbb{R}$ be a $\Gamma$-invariant $\mu$-measurable function. By Corollary 8.7, $\phi$ is $\mathcal{H}$-invariant. By Corollary 4.4 and Proposition $4.2, \phi$ is constant almost everywhere. Our theorem follows.

Acknowledgements. W. Goldman and S. Lawton were partially supported by U.S. National Science Foundation grants DMS 1107452, 1107263, 1107367 and 1309376 'RNMS: Geometric structures and representation varieties' (the GEAR Network). S. Lawton acknowledges support from U.S. National Science Foundation grant DMS 1309376 and also DMS 0932078000 while he was in residence at the Mathematical Sciences Research Institute in Berkeley, California during the Spring 2015 semester. He was also partially supported by a Simons Foundation Collaboration grant. E. Xia was partially supported by the Ministry of Science and Technology Taiwan with grants 1032115-M-006-007-MY2, 105-2115-M-006-006, 106-2115-M-006-008 and 107-2115-M006-009 and the National Center for Theoretical Sciences, Taiwan. 


\section{REFERENCES}

[BR89] U. Bhosle and A. Ramanathan. Moduli of parabolic G-bundles on curves. Math. Z. 202(2) (1989), $161-180$

[BS15] M. Brin and G. Stuck. Introduction to Dynamical Systems. Cambridge University Press, Cambridge, 2015.

[DK00] J. J. Duistermaat and J. A. C. Kolk. Lie Groups (Universitext). Springer, Berlin, 2000.

[FL14] C. Florentino and S. Lawton. Topology of character varieties of Abelian groups. Topology Appl. 173 (2014), 32-58.

[FM12] B. Farb and D. Margalit. A Primer on Mapping Class Groups (Princeton Mathematical Series, 49). Princeton University Press, Princeton, NJ, 2012.

[Gol20] W. Goldman. Parallelism on Lie groups and Fox's free differential calculus. Characters in Low-Dimensional Topology (Centre de Recherches Mathématiques Proc.) (Contemporary Mathematics, 760). Eds. O. Collin, S. Friedl, C. Gordon, S. Tillmann and L. Watson. American Mathematical Society, Providence, RI, 2020, to appear.

[Gol84] W. M. Goldman. The symplectic nature of fundamental groups of surfaces. Adv. Math. 54(2) (1984), 200-225.

[Gol86] W. M. Goldman. Invariant functions on Lie groups and Hamiltonian flows of surface group representations. Invent. Math. 85(2) (1986), 263-302.

[Gol97] W. M. Goldman. Ergodic theory on moduli spaces. Ann. of Math. (2) 146(3) (1997), 475-507.

[Got49] M. Gotô. A theorem on compact semi-simple groups. J. Math. Soc. Japan 1 (1949), 270-272.

[GP10] V. Guillemin and A. Pollack. Differential Topology. AMS Chelsea, Providence, RI, 2010.

[GX11] W. M. Goldman and E. Z. Xia. Ergodicity of mapping class group actions on SU(2)-character varieties. Geometry, Rigidity, and Group Actions (Chicago Lectures in Mathematics). University of Chicago Press, Chicago, IL, 2011, pp. 591-608.

[Hue95] J. Huebschmann. Symplectic and Poisson structures of certain moduli spaces. I. Duke Math. J. 80(3) (1995), 737-756.

[KH95] A. Katok and B. Hasselblatt. Introduction to the Modern Theory of Dynamical Systems (Encyclopedia of Mathematics and its Applications, 54). Cambridge University Press, Cambridge, 1995. With a supplementary chapter by Katok and Leonardo Mendoza.

[Law06] S. Lawton. SL(3, C)-character varieties and RP2-structures on a trinion. PhD Thesis, University of Maryland, College Park, ProQuest LLC, Ann Arbor, MI, 2006.

[Law07] S. Lawton. Generators, relations and symmetries in pairs of $3 \times 3$ unimodular matrices. J. Algebra 313(2) (2007), 782-801.

[OV90] A. L. Onishchik and È. B. Vinberg. Lie Groups and Algebraic Groups (Springer Series in Soviet Mathematics). Springer, Berlin, 1990. Translated from the Russian and with a preface by D. A. Leites.

[PX02] D. Pickrell and E. Z. Xia. Ergodicity of mapping class group actions on representation varieties. I. Closed surfaces. Comment. Math. Helv. 77(2) (2002), 339-362.

[PX03] D. Pickrell and E. Z. Xia. Ergodicity of mapping class group actions on representation varieties. II. Surfaces with boundary. Transform. Groups 8(4) (2003), 397-402.

[Wal82] P. Walters. An Introduction to Ergodic Theory (Graduate Texts in Mathematics, 79). Springer, New York, 1982. 